\title{
DSpace@MIT
}

\author{
MIT Open Access Articles
}

Locality-oblivious cache organization leveraging single-cycle multi-hop NoCs

The MIT Faculty has made this article openly available. Please share how this access benefits you. Your story matters.

Citation: Woo-Cheol Kwon, Tushar Krishna, and Li-Shiuan Peh. 2014. Locality-oblivious cache organization leveraging single-cycle multi-hop NoCs. SIGPLAN Not. 49, 4 (February 2014), 715-728.

As Published: http://dx.doi.org/10.1145/2541940.2541976

Publisher: Association for Computing Machinery (ACM)

Persistent URL: http://hdl.handle.net/1721.1/90541

Version: Author's final manuscript: final author's manuscript post peer review, without publisher's formatting or copy editing

Terms of use: Creative Commons Attribution-Noncommercial-Share Alike 


\section{Locality-Oblivious Cache Organization leveraging Single-Cycle Multi-Hop NoCs}

\author{
Woo-Cheol Kwon \\ Department of EECS, MIT \\ Cambridge, MA 02139 \\ wckwon@csail.mit.edu
}

\author{
Tushar Krishna * \\ Intel Corporation, VSSAD \\ Hudson, MA 01749 \\ tushar.krishna@intel.com
}

\author{
Li-Shiuan Peh \\ Department of EECS, MIT \\ Cambridge, MA 02139 \\ peh@csail.mit.edu
}

\begin{abstract}
Locality has always been a critical factor in on-chip data placement on CMPs as accessing further-away caches has in the past been more costly than accessing nearby ones. Substantial research on locality-aware designs have thus focused on keeping a copy of the data private. However, this complicates the problem of data tracking and search/invalidation; tracking the state of a line at all on-chip caches at a directory or performing full-chip broadcasts are both non-scalable and extremely expensive solutions. In this paper, we make the case for Locality-Oblivious Cache Organization (LOCO), a CMP cache organization that leverages the on-chip network to create virtual single-cycle paths between distant caches, thus redefining the notion of locality. LOCO is a clustered cache organization, supporting both homogeneous and heterogeneous cluster sizes, and provides near single-cycle accesses to data anywhere within the cluster, just like a private cache. Globally, LOCO dynamically creates a virtual mesh connecting all the clusters, and performs an efficient global data search and migration over this virtual mesh, without having to resort to full-chip broadcasts or perform expensive directory lookups. Trace-driven and full system simulations running SPLASH-2 and PARSEC benchmarks show that LOCO improves application run time by up to $44.5 \%$ over baseline private and shared cache.
\end{abstract}

Categories and Subject Descriptors $\quad$ C.1.2 [PROCESSOR ARCHITECTURES]: Multiple Data Stream Architectures (Multiprocessors)

\footnotetext{
* This work was carried out while Tushar Krishna was a graduate student at MIT
}

Permission to make digital or hard copies of all or part of this work for personal or classroom use is granted without fee provided that copies are not made or distributed for profit or commercial advantage and that copies bear this notice and the full citation on the first page. Copyrights for components of this work owned by others than the author(s) must be honored. Abstracting with credit is permitted. To copy otherwise, or republish, to post on servers or to redistribute to lists, requires prior specific permission and/or a fee. Request permissions from permissions@ acm.org.

ASPLOS '14, March 1-5, 2014, Salt Lake City, Utah, USA.

Copyright is held by the owner/author(s). Publication rights licensed to ACM. ACM 978-1-4503-2305-5/14/03 .. \$15.00. http://dx.doi.org/10.1145/2541940.2541976
Keywords Multiprocessor; CMP Cache Design; Cache Coherence; Locality; Network-On-Chip

\section{Introduction}

Chip Multi-Processors (CMPs) have become mainstream in recent years, providing increased parallelism as core counts scale. While a tiled CMP with a mesh Network-on-Chip (NoC) is widely accepted to be a scalable architecture for the many-core era [21, 22, 39, 43], on-chip cache organization and coherence are far from solved problems.

Private and shared LLCs (Last Level Caches) form two extremes of the design spectrum for cache organization. Private caches offer the fastest access to data $(\sim 10-20$ cycles for L2) but lead to overall reduction in cache capacity due to replication, and make invalidations expensive (via broadcasts or precise multicasts if all sharers are tracked). Shared caches offer the best utilization of cache capacity, but every access becomes remote, bringing in on-chip network delay which conventionally increases as hop count scales with core count. Almost all works on scalable cache organization try to mimic the capacity of a shared cache and the performance of a private cache. Prior approaches fundamentally boil down to keeping data as close as possible to the requesting core, like a private cache, but minimizing unnecessary replication. These Locality-Aware approaches include optimized page placement [3,10,14, 15, 19], line placement [12], intelligent replication $[7,13,46]$ and clustering $[9,18,31,35]$.

However, we believe that the locality-aware approach is not just hard (ask any software/OS developer!), but also fighting a losing battle since private cache capacity is not expected to increase much while applications are continuously becoming more heavily multi-threaded with lots of sharing and larger data sets. Current commercial designs, such as Intel's latest Xeon 5500 Series use a single homogeneous chunk of 8MB L3 cache shared by 4-8 cores which takes 4075 cycles to access. Having a large many-ported central LLC will lead to high delay, area and power penalties, and hence the approach has been to physically distribute LLC banks across tiles [7, 13, 30, 46]. At 256 cores, distributing a 16MB LLC will lead to only $64 \mathrm{kB}$ chunks per tile. Moreover, com- 


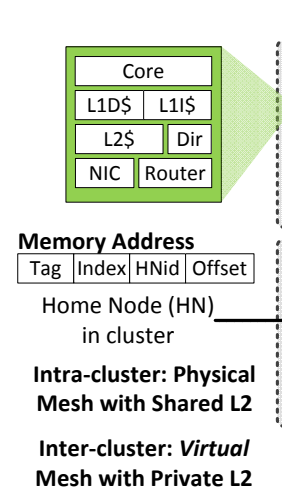

Mesh with Private L2
Cluster Size $=4 \times 4$

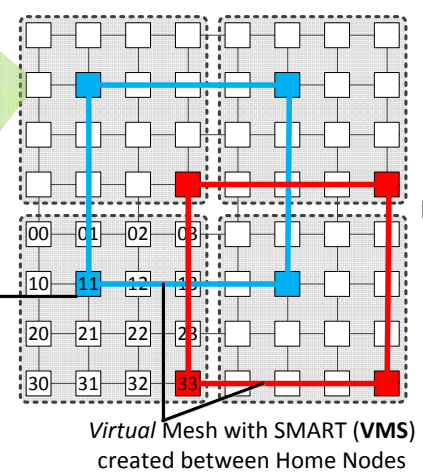

Cluster Size $=4 \times 1$

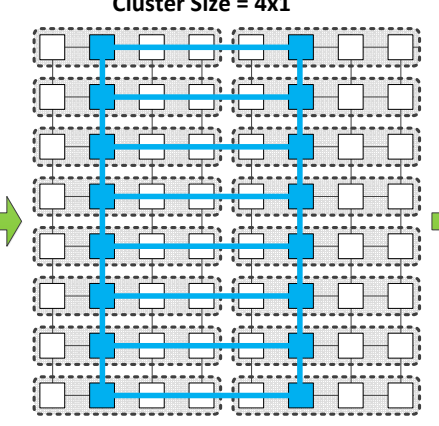

1-2 cycle traversal within and across clusters using SMART NoC
Heterogenous Clusters

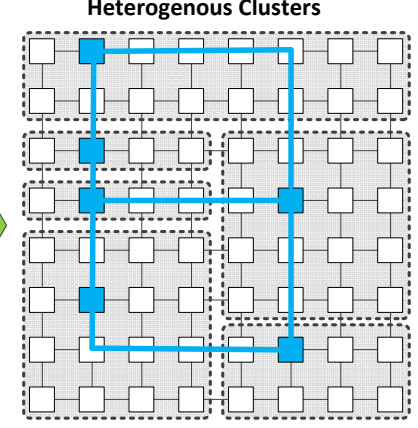

Figure 1: Overview of LOCO plex locality-aware approaches tend to complicate the cache coherence protocol by adding more states and indirection to the already non-scalable directory $[17,30,32,42]$ to track these "intelligently" placed lines on-chip. Global data search via full-state directories, or full-chip broadcasts are both non-scalable and expensive. Hierarchical (clustered) cache designs mitigate the tracking problem since the directory can track lines at the cluster rather than core granularity. However, data search now requires multiple indirections (search within local cluster $\rightarrow$ indirection to directory $\rightarrow$ forward to remote cluster $\rightarrow$ search within remote cluster $\rightarrow$ response).

The underlying premise behind all locality-aware approaches is the conventional wisdom that accessing a remote cache is expensive. Thus these designs actively optimize both the cache allocation and replacement algorithms to keep copies of data being used by the core in the local (private) cache, and to minimize useless replicas that hog up precious cache space. In this paper, we challenge this assumption, and show that on-chip networks can be designed in today's (and future) technology to realize a single-cycle access to a remote $\mathrm{L} 2$, essentially killing the motivation for keeping data private in the first place.

We introduce Locality-Oblivious Cache Organization (LOCO). LOCO is a co-design of the on-chip network and cache-coherence protocol to support a hierarchical, flexible private-shared cache organization. An overview is shown in Figure 1. LOCO groups cores into virtual clusters of any size, sharing a (distributed) chunk of the LLC. Within each cluster, there can be only one copy of data. We leverage a recently proposed network-on-chip (NoC) design called SMART(Single-cycle Multi-hop Asynchronous Repeated Traversal), that dynamically creates multi-hop paths in the network and traverses messages within a single-cycle by using clockless drivers at each router. SMART enables near single-cycle access to the home node within each cluster. LOCO creates a Virtual Mesh with SMART(VMS) between the home nodes of each cluster, and allows data replication across the home nodes. Efficient global searches are performed across the chip by multicasting over the VMS, instead of full-chip broadcasts or expensive full-bit directories. Creating virtual topologies rather than using fixed, physical ones provides immense flexibility in choosing the cluster size at running applications, rather than at design time. To increase effective on-chip capacity, LOCO allows evicted data from one cluster to migrate to another, without adding any complexity to the coherence protocol since there is no directory tracking the lines across clusters that needs to be updated. This is in contrast to prior work which has looked at cache line migration [16] which tends to complicate the coherence protocol and directory to support migration.

Evaluation results show that LOCO provides much superior performance over private and shared cache. For 64 cores, full system simulation shows that LOCO reduces application run time by $44.5 \%$ on average. In trace-driven simulation conducted for both 64 and 256 cores, LOCO shows scalability to higher core counts, giving $17.9 \%$ improvement for 256 cores, while the run time reduction for 64 cores is $13.9 \%$. Further, we show that LOCO can give higher performance by providing adaptive cluster size according to application characteristics.

The rest of this paper is organized as follows. Section 2 presents relevant background on SMART NoCs and the underlying technology. Section 3 presents our LOCO design. Section 4 reports experimental results. Section 5 reviews related work, and Section 6 concludes this paper.

\section{Background: SMART NoC}

A key enabler for LOCO is a network that allows singlecycle traversals, thereby making an access to a remote $\mathrm{L} 2$ no worse than an access to the local (in tile) L2 within a cluster. What limits us from designing such single-cycle networks today? It turns out that it is not the wires themselves. Global wires (i.e. wires connecting two tiles) with repeaters have been shown to traverse 10-16mm within 1ns [11, 20, 25, 29] at technologies today. Assuming a tile-size of $1 \mathrm{~mm}$, this translates to 10 to 16 hops at a $\mathrm{GHz}$ clock. As technology scales, this delay is expected to remain similar ${ }^{1}$ [29]. This trend of wires not becoming faster with scaling has often

\footnotetext{
${ }^{1}$ Intuitively, as the feature size goes down, wires become thinner so their resistance goes up, while their capacitance goes down (assuming wires are kept at 2.5-3 times the minimum pitch in that technology to mitigate effects of coupling capacitance), keeping wire delay approximately the same.
} 
been considered a thorn in the flesh, and has been the motivation for most locality-aware designs. But we argue that this trend does not hurt us since chip dimensions are expected to remain similar as well $(\sim 20 \mathrm{~mm} \times 20 \mathrm{~mm})$ due to yield, and clock frequencies are going to remain constant due to the power wall. If we couple these two observations with the trend of same wire delay and smaller tile sizes (with scaling), we can conclude that future technologies will allow wires to traverse even more hops within a cycle, making a compelling case for locality-oblivious designs.

But wire delay is not the end of the story. Even if wires can allow cross-chip traversals at a $\mathrm{GHz}$, the limiter is the on-chip network, which multiplexes multiple flows over the same wire segments, forcing a hop-by-hop traversal via routers. The best router designs today [38] have been able to reduce arbitration delay for the crossbar and wire segment to a single-cycle, leading to 2 cycles per hop (one in the router, and one on the link). But this still does not help if the data to be fetched is many hops away on the chip. Adding 1-cycle dedicated wires [25] across distant routers [28] has been one approach for reducing network delay, but this comes with the exorbitant cost of multi-stage arbiters and crossbars at each multi-port router, thus forcing practical designs to make do with few physical express links which then pay off only if the traffic maps well to the topology.

SMART (Single-cycle Multi-hop Asynchronous Repeated Traversal) [29] is a recent NoC architecture that builds virtual single-cycle multi-hop paths within the network. SMART can be overlaid on any topology, but throughout this paper, we will assume a mesh topology. The reason why we use SMART, and not a physical express topology like Flattened Butterfly [28] with LOCO is to enable us to create virtual meshes over varying cluster sizes. Here, we briefly explain how SMART NoCs work. We will discuss how we extend SMART for efficient broadcasts over LOCO's VMS in Section 3.2.

SMART replaces conventional clocked drivers on each router-to-router link by clockless repeaters ${ }^{2}$. This is shown in Figure 2a. Now, flits can traverse multiple hops within a cycle, latched only at the final hop. Whether a flit is buffered at intermediate routers is controlled by the buffer enable and crossbar select signals, which are preset one cycle before the flit arrives. The maximum number of hops that can be traversed within a cycle is a design time parameter called $H P C_{\max }$ (Hops Per Cycle) which will be described later.

A typical network traversal is shown in Figure $2 \mathrm{~b}$. At every router, the winner of the arbitration for the switch's output port broadcasts a SMART Setup Request (SSR) up to $H P C_{\max }$ hops via dedicated SMART links. The SSR carries the length (in hops) up to which the flit wishes to go.

\footnotetext{
${ }^{2}$ As for the energy consumption, clockless repeaters require $14.3 \%$ lower energy than clocked drivers, but there is additional overhead due to multihop data-path and SSR signaling, leading to similar overall energy to conventional NoC [29].
}

For instance, in Figure 2b, $S S R_{A}=3$ indicates a 3-hop path request from Flit $A$. Each router arbitrates among the SSRs that it receives, giving higher priority to local/nearer flits over farther flits, and sets up the buffer enable, and crossbar select signals appropriately. In the next cycle, the actual flits traverse the switches and links, bypassing intermediate nodes, potentially going all the way through $H P C_{\max }$ hops before stopping and being buffered. This process of sending SSRs that pre-set routers a cycle before flits arrive continues until the flit arrives at its destination.

We define SMART-hop to be 「hops_to_dest/HPC $\left.C_{\max }\right\rceil$, i.e. the minimum number of multi-hop single-cycle traversals required to reach the destination. The best case latency for a SMART-hop is 2 cycles (SSR followed by ST-LT) ${ }^{3}$. But it could be more depending upon contention because a flit could be stopped prematurely, before it completes its SMART-hop, and/or because it could take multiple cycles within the router before it wins the switch. Figure $2 \mathrm{c}$ illustrates an example of contention, where Router 31 arbitrates between the SSRs of Flit A and Flit B, prioritizing the latter to use the output link, and prematurely buffering the former. In the next cycle, Flit A will arbitrate for the local switch, and should it win, continue its traversal by sending a fresh SSR.

In this work, we use the simpler SMART_1D design [29] which does not allow bypassing at turns, i.e. turning flits have to stop at the turning router, and send fresh SSRs for the other dimension ${ }^{4}$. Thus an X-only and Y-only traversal takes at least one SMART-hop, while an X+Y traversal takes at least two SMART-hops as shown in Figure 2b. Each SMART-hop takes 2-cycles in the best case. Going from one corner of a $8 \times 8$ mesh to the opposite corner (i.e. 14 hops), with $H P C_{\max }=4$, takes 4 SMART-hops, i.e. 8 cycles in the best case. In a conventional NoC, it would take 28 cycles in the best case.

Derivation of $\mathbf{H P C}_{\max }$. A test chip at $45 \mathrm{~nm}$ recently demonstrated that clockless repeated links can go up to $13 \mathrm{~mm}$ with $1.0 \mathrm{~V}$ full-swing ( $16 \mathrm{~mm}$ with $300 \mathrm{mV}$ low-swing) within 1ns [11]. Post-layout simulations of the synthesized SMART control and data paths in $45 \mathrm{~nm}$ indicate that SSR traversal followed by arbitration limits the speed to $9-11 \mathrm{~mm} / \mathrm{ns}$ [29]. In this work we choose $8 \mathrm{~mm} / \mathrm{ns}$-sized repeaters (to allow us to size our clusters as a power of 2 (Section 3.1). At $2 \mathrm{GHz}$, for $1 \mathrm{~mm} \times 1 \mathrm{~mm}$ tiles, this gives us a $H P C_{\max }$ of 4.

\footnotetext{
${ }^{3}$ The best case latency for a conventional state-of-the-art $\mathrm{NoC}$ router is also 2 cycles per hop [38] as described earlier. Thus a conventional NoC will take $2 \times H P C_{\max }$-cycles to cover a SMART-hop in the best case.

${ }^{4}$ This is because SMART_1D was shown to be good enough to achieve most of the performance benefits of SMART for LOCO, without the complexity of incorporating bypass at turns.
} 


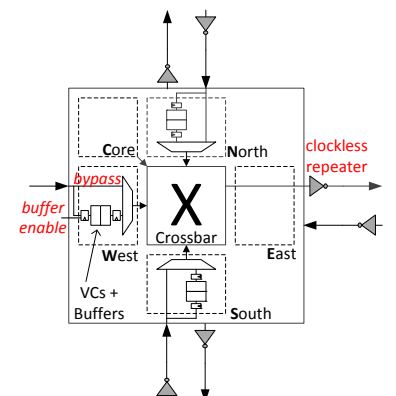

(a) SMART Router Microarchitecture

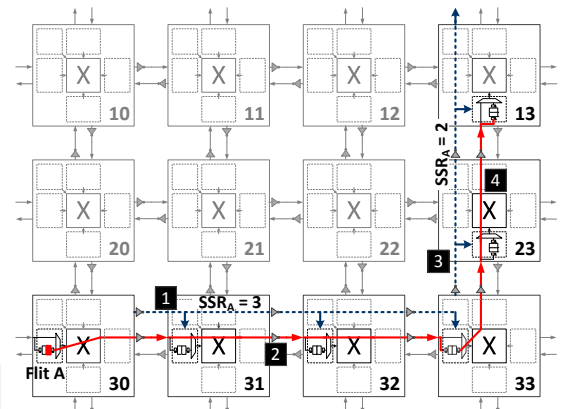

(b) XY Traversal for Flit A with no contention.

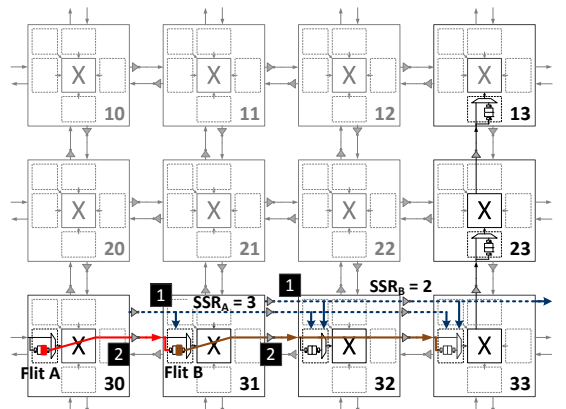

(c) Flit A prematurely stopped at Router 31 due to contention with Flit B.

Figure 2: SMART: Single-cycle Multi-hop Asynchronous Repeated Traversal [29]

\section{Locality-Oblivious Cache Organization}

\subsection{Local Cache Clustering}

LOCO proposes a two-level hierarchical approach for L2 cache organization. At the local level, it first partitions the CMP into multiple cache clusters. The clusters can be 1D or 2D meshes of any size, as shown in Figure 1. The size of the cluster depends on the applications' working sets and/or aggregate cache requirement [18, 23, 31, 35], and is not the focus of this work. However, we do believe that $H P C_{\max }$ should drive the cluster sizes, since any cache within $H P C_{\max }$ hops can typically be accessed in 1 (X-only or Y-only) to $2(\mathrm{X}+\mathrm{Y})$ SMART-hops (which corresponds to 2 to 4 cycles low-load latency), as explained in Section 2. Cluster sizes less than $H P C_{\max }$ do not buy us much since they only result in more replication without lowering access delay.

Within a cluster, the organization of L2 cache slices is like that of a distributed shared cache [4] where the mapping of data to L2 cache slice is statically determined given the address. As shown in Figure 1, the least significant bits of a block address (HNid) are used to identify the home node for fair load balancing as is usually done in shared caches.

Several prior works have proposed fully flexible data mapping to L2 slices, so that the first requestor of data becomes the home node of the data $[14,19,45]$. These approaches aim at minimizing L2 access latency by placing data into the local L2 cache in the same tile as the requesting core, which is especially beneficial when the majority of data is local (accessed by only one core). In contrast, LOCO employs static data mapping by address. The rationale behind this is that, via SMART links, properly sized local clusters provide low latency to any L2 slice inside the cluster. With the width and height of the cluster set within $H P C_{\max }$, accessing any L2 slice and back mostly takes only 4-8 cycles in the network, which is of the same order as typical private L2 access latencies. Fixed data mapping also allows only one data copy inside a cluster, which leads to higher cache hit rate by increasing effective cache capacity. Hence, LOCO leverages SMART NoC to ensure fast L2 cache ac- cess within a cluster, while delivering a larger shared cache that overcomes the lower cache hit rate of small individual private caches.

Static data mapping within a cluster has an additional advantage over flexible mapping - when searching for data in the other clusters, it suffices to check only one candidate L2 per cluster. Moreover the locations are known a priori by the address. As a result, static data mapping significantly reduces the search space, and thus facilitates an efficient global data search strategy over the entire chip. We will present this strategy in the next section.

In a distributed shared cache with inclusive L2, any cache request should first access the L2 cache slice at the home node. As a result, a directory-based protocol is a natural choice for maintaining cache coherence within LOCO clusters. Directories at each home node keep track of sharers only from the local cluster, not across the entire chip. This, in turn, ensures that the storage overhead for directory is manageable.

\subsection{Global Data Search}

At the global level, LOCO operates each cluster like a large private cache. When the first requesting core receives data from off-chip memory, the data is stored in its local cluster. Subsequent requestors search for the data on-chip and replicate them in their own local clusters for fast future look-up.

To realize fast access to data in other clusters, LOCO adopts a broadcast protocol to maintain cache coherence between clusters. Unlike a directory-based counterpart, a broadcast protocol sends requests directly to all possible sharers, thereby saving indirection latency in looking up a tag directory.

However, broadcasting over the entire chip does not scale to high core counts, as power consumption and bandwidth demand grow prohibitively costly with increasing number of cores. To tackle this scalability problem, we take advantage of the nice topological property that LOCO's cluster organization offers. As already mentioned, the home node location within a cluster is fixed by the address. We can create a Virtual Mesh with SMART links(VMS) between these home 
nodes. Since SMART creates virtual multi-hop bypass paths, different cluster sizes/shapes can result in different VMS sizes/shapes. Figure 1 shows two $4 \times 4$ VMSs, for HNid=11 and $\mathrm{HNid}=33$, in a 64-core system. The highlighted routers on each VMS are called home routers, while all other routers along the route are called intermediate routers. Flits only stop at the home routers, and try to bypass all intermediate routers by creating single-cycle multi-hop paths between home routers.

LOCO broadcasts a global cache requests only on a specific VMS (based on the address), avoiding costly broadcasts to the entire chip. While the original SMART design only supported unicasts, we extend it to support broadcasts over VMSs. We modify two components in each router: route computation and switch arbitration. Each broadcast uses an $\mathrm{XY}$-tree multicast routing algorithm, connecting all nodes in the VMS into a tree, as shown in Figure 3 for the VMS with $\mathrm{HNid}=11$. At each home router, including the starting home router, the flit requests for one or more output ports depending on its location on the tree (which can be identified by its input port). For example, in the highlighted router in Figure 3, the flit enters the home router at the East input port, and places a request for the West, North, South and Ejection output ports. Each output port arbitrates independently, and the flit can be granted one or more output ports depending on contention. Each output port winner then sends out SSRs, just like in the baseline SMART, except that a broadcast triggers simultaneously SSRs across multiple directions, with the SSR always set to $H P C_{\max }$ (4 in this design), indicating a request to stop at the next home router on the VMS. In the next cycle, the flit performs ST-LT across multiple hops along one or more directions. Forking of flits does not stretch timing because select lines of the crossbar muxes automatically fork the flit, and each output link has its own repeater. In Figure 3, the flit completes the broadcast in 4 SMARThops, i.e., 8 cycles in the best case ${ }^{5}$. Without this broadcast extension to SMART, 15 copies of the flit would have to be created and sent from the source as unicasts, increasing contention at routers, adding to network delay.

\subsection{Inter-Cluster Victim Replacement (IVR)}

To maximize usage of precious on-chip cache capacity, LOCO leverages other clusters for victim caching. On any L2 eviction at the cluster home node, a replacement request is sent to the corresponding home node in a randomly chosen cluster, with the timestamp of its last access. Timestamps are approximations, implemented by incrementing a counter every $T$ cycles to reduce area overhead. On receiving this

\footnotetext{
${ }^{5}$ The best case occurs when there is no contention in establishing SMART paths in the network. In the worst case, the flit might stop at every intermediate router, which results in 32 cycles, the delay of a conventional network with a single-cycle router pipeline. Network contention can further aggravate the delay, forcing the flit to waste more cycles at each router waiting to win arbitration. This downside of virtual SMART paths (compared to physical express links) is modeled in our simulations.
}

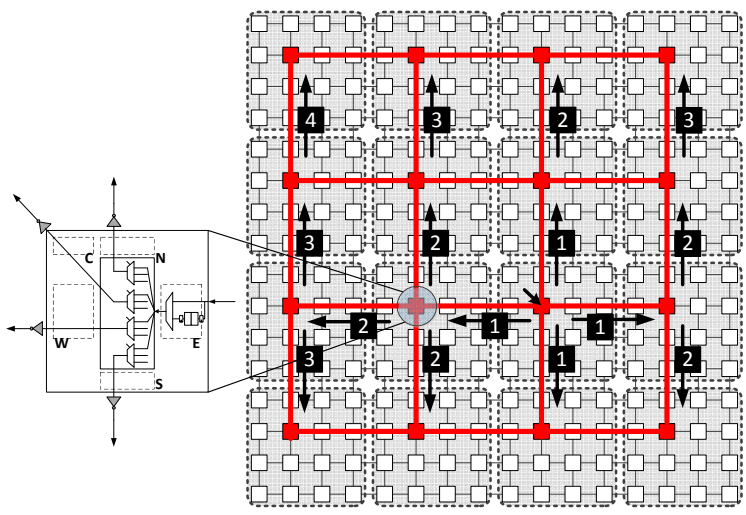

Figure 3: Example broadcast over a VMS

replacement request, the remote home node selects a local victim, and compares the timestamp of its victim to that of the migrating data. The cache line with the older timestamp is chosen to be the final victim, and is evicted to other clusters for another replacement attempt. Choosing target clusters randomly ensures balanced utilization of L2 cache resource across the chip.

IVR effectively extends the reach of one cluster into others. A cluster can retrieve its data stored in other clusters in very few cycles, owing to the fast global data search explained in the previous section. IVR enables cores that heavily use caches to offload to relatively underutilized clusters, since evicted cache data from more cache demanding cores are likely to have more recent timestamps.

We avoid deadlock by prohibiting the L2 cache from waiting for the outgoing queue to the network during IVR. If the L2 cache finds that the outgoing queue is full, it sends the victim date directly to the off-chip memory via the write-back virtual network. There is also a potential livelock scenario in which the evicted data with the oldest timestamp loops in the on-chip system endlessly. To prevent this, we add a replacement counter in the header flit of the migrating victim data. When this counter hits a certain threshold (4 is assumed in this design), the data is sent back to off-chip memory.

\subsection{Walkthrough Examples}

The example system is a 64-core CMP employing a $8 \times 8$ mesh network, which is partitioned into 4 fixed-size local clusters, each comprising $4 \times 4$ tiles; the entire system consists of $2 \times 2$ local clusters. For our example, we assume hierarchical L1/L2 cache organization with inclusive L2 cache. L1 cache has MSI states for cache coherence, while L2 cache has MOESI states. We will indicate the specific coherence state for L1 or L2 caches by State:L1 or L2 respectively.

Figure $4 \mathrm{a}$ shows the steps involved in a cache hit. (1) Node C, initially in I:L1 state, sends a READ request to local home node H. (2) H, in S:L2 state, returns the data to the requestor. When a cache hit occurs, the request is handled within the cluster by a directory-based protocol, tracking the 16 potential sharers of the cluster. Hence, with no network 


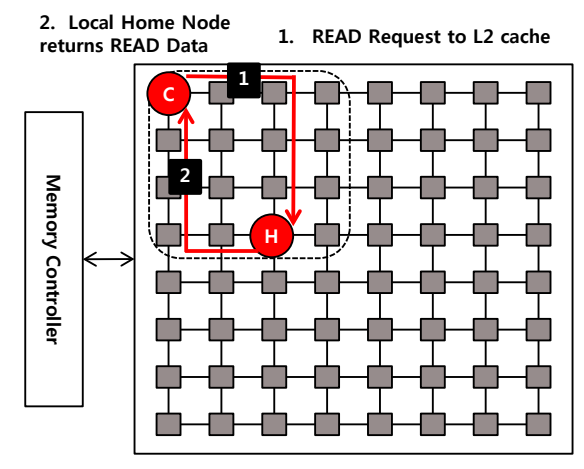

(a) Cache Hit Example

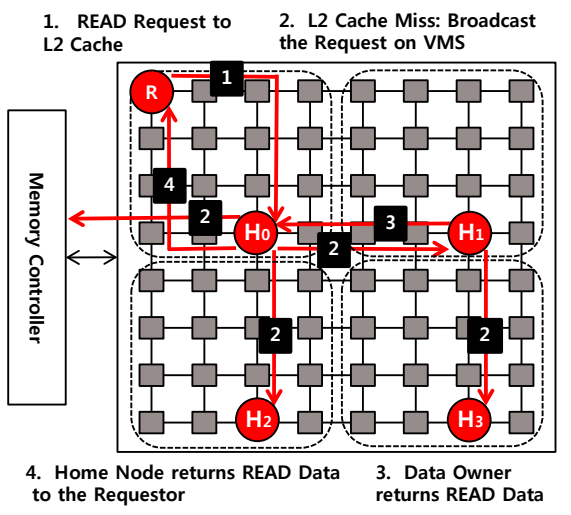

(b) READ Miss Example

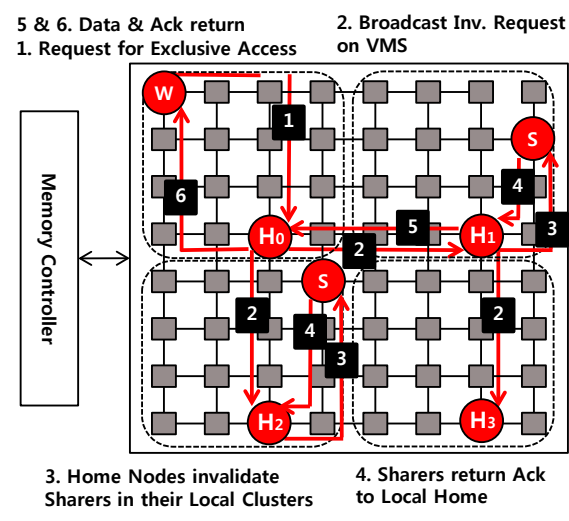

(c) WRITE Example

Figure 4: Walkthrough Examples

contention, a SMART path enables L2 cache hits to take only 4 cycles at most. Figure $4 \mathrm{~b}$ shows a case where the READ request results in a cache miss. (1) The request sent from $R$ arrives at its local home node $H_{0}$. (2) $H_{0}$, in I:L2 state, finds no matching tag entry at its L2 cache. Now it invokes the second level protocol by broadcasting the request to other clusters on the VMS for the address. The request is delivered to $H_{1}, H_{2}$, and $H_{3}$ along SMART links. The request is sent to off-chip memory as well. (3) To ensure only one sharer returns the data among multiple shares, the one with ownership, i.e. in $\mathrm{O}$ state $\left(H_{1}\right.$ in our example) responds to the request. For simplicity, this example only shows the case where the data is cached on-chip. If there is no cached data on-chip, the memory controller assumes the ownership and returns the data from off-chip memory. (4) Finally, $H_{0}$ stores the data into its L2 cache and transits to S:L2 state. Now it goes back to the first level protocol, and returns the data to the requestor, $R$.

Next, we turn to WRITE operations in Figure 4c. (1) Node $W$, in I:L1 state, issues the request for exclusive access to its local home node $H_{0}$. (2) To get exclusive access right, $H_{0}$ broadcasts invalidation requests on the VMS. (3) Home nodes in each cluster invalidate their local sharers by the first level protocol. Each home node checks a tag directory to retrieve the local sharer list and invalidates them. (4) Local sharers in S:L1 state transit to I:L1 state, and send acknowledgements to local home nodes. (5) After invalidating local sharers, home nodes also transit to I:L2 state. In this example, $H_{1}$ was in O:L2 state. It sends the data back to the home node of the requesting cluster, $H_{0}$. On receiving the data, $H_{0}$ changes its state to M:L2 state. (6) $W$ finally receives the data, and transits to M:L1 state.

Next we will walk through inter-cluster victim replacement in Figure 5. (1) A new READ request causes cache replacement at the local home node $H_{0}$. (2) $H_{0}$ selects a victim cache line and sends it to a random destination $H_{1}$ (3) $H_{1}$ receives the replacement request, and selects a local victim cache line via timestamp comparison. If the timestamp of the migrated data is newer, $H_{1}$ stores the migrated

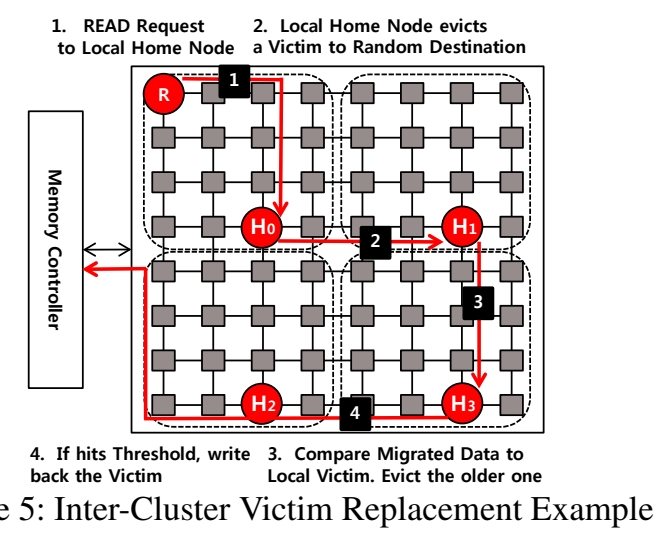

Figure 5: Inter-Cluster Victim Replacement Example

data in its L2 cache and evicts its local victim, migrating it to a random destination, repeating the entire process. Otherwise, replacement is denied, and instead the data is steered to another random node. (4) $H_{3}$ receives the victim data and finds that the replacement counter has hit the threshold. It sends the data as write-back to the off-chip memory.

\section{Evaluation}

We implemented LOCO and the underlying SMART NoC (extended for efficient VMS broadcasts) in the C++-based multi-core simulation tool GEMS [34], which incorporates the cycle-accurate network model GARNET [1].

We perform trace-driven simulations for both 64-core and 256-core CMPs by feeding traces generated by Graphite $[37]^{6}$ into GEMS. We also verify LOCO with full-system simulations to provide more realistic evaluation results. As fullsystem simulations on GEMS lead to unfeasibly long simulation time beyond 64 cores, full-system simulations were conducted only for 64-core CMP to maintain the simulation time within a week.

We ran SPLASH-2 [44] and PARSEC [8] benchmark suites. Statistics are gathered at the end of the parallel por-

\footnotetext{
${ }^{6}$ While Graphite provides fast, scalable full-system simulation up to 1000 cores, it does not at the moment provide a cycle-accurate network model yet, which is crucial for correct evaluation of LOCO.
} 
tion of each benchmark. Simulations run to completion for the 64-core full-system simulations. However, due to simulation time limitation, our trace-driven simulations simulate up to 2 billion memory instructions including cache warmup sequences.

Table 1: Target System Configuration

\begin{tabular}{|c|c|}
\hline \multicolumn{2}{|r|}{ Processing Tile } \\
\hline Processing cores & 2-way in-order SPARC \\
\hline Cache line size & 32 Bytes \\
\hline L1 cache & Split 16KB I\&D, 4-way, 1-cycle access latency \\
\hline L2 cache & $\begin{array}{l}\text { Inclusive unified 64KB per each tile } \\
8 \text {-way, 4-cycle access latency }\end{array}$ \\
\hline Cache coherence & MSI for L1 Cache, MOESI for L2 Cache \\
\hline \multicolumn{2}{|r|}{ On-Chip Network } \\
\hline Topology & $8 \times 8$ mesh for 64 -core, $16 \times 16$ mesh for 256 -core \\
\hline Routing & XY dimension-ordered \\
\hline Virtual Networks (VNs) & 5 \\
\hline Virtual Channels per VN & 4 \\
\hline Link channel width & 16 Bytes \\
\hline$H P C_{\max }$ for SMART & 4 \\
\hline \multicolumn{2}{|r|}{ Memory Interface } \\
\hline Directory & 10-cycle access latency \\
\hline Memory & $\begin{array}{l}4 \text { memory controllers (one on each edge) } \\
200 \text {-cycle access latency }\end{array}$ \\
\hline
\end{tabular}

\subsection{Target CMP System}

Our target system consists of strictly hierarchical L1/L2 caches. L1 cache is allowed to communicate only with L2 caches, not directly with other L1 caches. We compare LOCO with two baseline cache organizations: distributed private and shared caches. Both baseline cache organizations and LOCO naturally get performance benefits from SMART NoCs, since all multi-hop traversals can now take just a single cycle. By running all cache organizations over SMART NoCs, we seek to highlight that the performance improvement over the baselines is strictly due to LOCO.

Cache Coherence. The baseline configurations operate on a directory-based protocol. When a L1 cache miss occurs, the request is delivered to the local L2 or the static home node in the shared and private L2 respectively. Then, the L2 cache communicates with other L2 caches or memory controllers to retrieve data from the sharers or to invalidate all shared data copies. The sharer information of each cache line is stored in a directory at the home node (shared cache) or at the memory controller (private cache).

LOCO uses $4 \times 4$ clusters (Figure 1), unless specified. We use directory-based coherence within clusters, and a broadcast protocol between clusters. Broadcast protocols assume an ordered interconnect, but LOCO's virtual meshes (VMS) do not provide ordering. As there have been recent proposals such as Token coherence [33] and INSO [2] for maintaining global ordering on a mesh topology, we can apply either one to LOCO. For our evaluations, we used Token Coherence, where a reader is required to have at least one token, while a writer must collect all tokens to obtain exclusive access right. With a L2 cache miss, the request is broadcast to all relevant L2 caches for the cache line address. On receiving

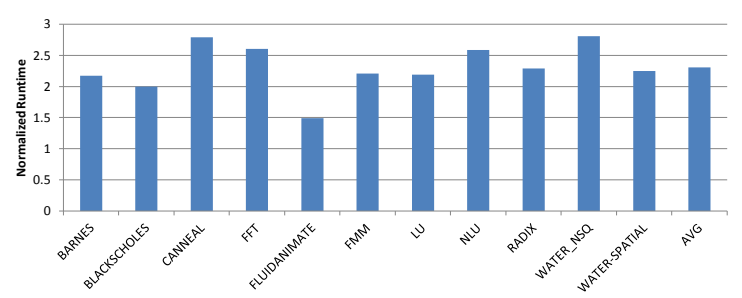

Figure 6: Normalized runtime of distributed private caches vs. distributed shared caches (64-core).

cache requests, L2 caches respond to the request by returning tokens or data.

Directory Storage and Latency Overhead. One important parameter impacting evaluation results is directory access latency. For baseline caches, the directory should cover sharers across the entire chip. A naive implementation based on a bit-vector, for instance, will require additional 256 bits per each cache line to keep track of sharers for a 256-core system, which is the same amount of storage needed for our 32-byte cache line. In contrast, a cluster-based approach like LOCO requires just a 16-bit vector for tracking sharers within the cluster. Directory storage overhead is one of key problems in maintaining scalability of directory-based protocols. There have been a plethora of suggestions such as sparse directory [17] or hierarchical directory [42] to mitigate directory storage overhead with a trade-off between performance and scalability. Addressing the scalability issues of a directory-based protocol is beyond the scope of this paper. Instead, we assume that the home node can access its directory in parallel with an L2 cache access without additional latency. Further we do not consider any adverse effect of directory storage overhead, which otherwise can be traded off for larger L2 cache capacity. This is a generous assumption for the baseline distributed shared cache organization. LOCO's inter-cluster broadcast protocol also needs a directory to record the ownership and related state bits, but it requires less storage overhead thanks to clustering.

\subsection{Evaluation Results: Trace-Driven Simulation}

To evaluate the performance of LOCO, we simulated three cache configurations: LOCO CC represents LOCO with just local cache clusters managed by a directory-based protocol providing global coherence. In LOCO CC, when a L2 cache miss occurs, the request is sent to the directory at the memory controller to obtain the sharer list. Next, it either receives a data copy from one of the sharers, or invalidates all sharers upon writes. It should be noted that the traversals to/from the directory are expedited through the SMART NoC as well. LOCO CC+VMS adds broadcasting on VMS between LOCO clusters. Finally, LOCO CC+VMS+IVR incorporates inter-cluster victim replacement as well.

Private vs Shared Cache. Figure 6 plots the runtimes of private caches, normalized to that of shared caches, both running atop SMART NoCs. It should be pointed out that baseline shared caches stand to gain more benefit from SMART 
NoCs as there is more global, cross-chip traffic compared to private caches. We observe that the performance of private caches is severely degraded due to high cache miss, on average 2.3 times slower than shared cache. This is due to small L2 cache slice $(64 \mathrm{~KB}),{ }^{7}$ assumed, which is arrived at given our assumption of small $1 \mathrm{~mm} \times 1 \mathrm{~mm}$ tiles in a manycore chip. From here onwards, we thus assume distributed shared caches as the baseline for comparison, unless stated otherwise.

L2 Cache Hit Latency. LOCO's clustering is designed to provide fast $\mathrm{L} 2$ cache access within a cluster, mimicking a large private L2 cache. Figure 7 shows the additional cache access latency of each cache configuration over that of a distributed private cache, i.e. the average latency of the baseline private cache is deducted from the other configurations.

For the 64-core CMP, as shown in Figure 7a, LOCO adds 2.9 cycle-latency on average for L2 cache access than private cache, while shared cache adds 11.5 cycles on average. Since each SMART-hop takes 2 cycles without network contention, LOCO requires just 1.5 more SMART-hop-delay than private cache, while the baseline shared cache needs additional 3.7-SMART-hop delay. In other words, since a baseline private caches takes 1-hop to send a request, and 1-hop to receive a response, LOCO takes just 3.5-SMART-hop delay on average.

As core counts increase, the benefit of LOCO also increases in comparison with shared cache. For 256-core CMP, as shown in Figure 7b, L2 access latency of shared cache increases by 4.5 cycles over 64-core CMP, while the latency of LOCO remains relatively unchanged with a marginal increase of 0.08 cycles.

L2 Cache Miss Rate. High cache hit rate is another goal of LOCO's clustering, enabled by the larger cache capacity of an entire cluster. Figure 8 shows L2 cache misses per a thousand instructions of various benchmarks, averaged across the entire simulation time. For 64-core CMP, LOCO increases L2 cache miss moderately over shared cache, by $0.33 \%$ for 64 -core CMP and by $0.43 \%$ for 256 -core CMP. Combined with L2 hit latency results above, this shows that LOCO comes reasonably close to our goal of offering low latency benefits of private cache while delivering the high cache hit rate of shared cache at the same time.

Impact of LOCO's Broadcast over Virtual Meshes. Figure 9 shows the cost involved in searching on-chip cached data with and without VMS, i.e. the average delay needed to find on-chip data stored in other clusters. This delay includes L2 cache access latency, directory latency, and network delay. In LOCO without VMS, the search is based on a directory protocol, which incurs indirection latency for directory look-up. As shown in the figures, VMS provide $34.8 \%$ reduction in search cost for 64 -core, and $39.9 \%$ for

\footnotetext{
${ }^{7}$ We note that we used very small-scale working sets in our simulations for tractability. In a real system, larger L2 caches will not result in significantly lower cache miss rates with realistic working sets.
}

256-core, respectively. This reduction is achieved via fast hardware broadcast support for VMS within SMART NoC routers, and by avoiding directory look-up latency. We note that VMS gives higher benefit for 256-core CMP with increased network delay.

Impact of LOCO's inter-cluster victim replacement. Another goal of LOCO is to effectively utilize on-chip cache capacity to reduce expensive off-chip memory access. LOCO $\mathrm{CC}+\mathrm{VMS}+\mathrm{IVR}$ is designed to address this. Figure $10 \mathrm{com}-$ pares the number of off-chip memory accesses of different cache configurations, normalized against the baseline shared cache. IVR shows $15.6 \%$ reduction of off-chip memory access over LOCO CC for $64-$ core, and $17.9 \%$ for 256 core. Overall, LOCO CC+VMS+IVR almost matches that of shared cache on average.

It is interesting to note that although shared cache has the largest effective cache capacity, in disallowing any redundancy across L2 cache slices, LOCO CC+VMS+IVR sometimes outperforms shared cache in reducing off-chip memory access, for example, barnes and swaptions for 64-core CMP. This is because LOCO CC+VMS+IVR extends the limited 8-way set-associativity of shared cache by migrating victims to other clusters. In a 64-core CMP, for instance, LOCO effectively creates a $8 \times 4=32$-way cache distributed across 4 distant clusters.

Overall Performance. Figure 11a shows normalized runtimes of each feature of LOCO progressively, against the baseline shared cache for 64-core CMP with trace-driven simulations. On average, $\mathrm{LOCO} C \mathrm{C}$ delivers $5.5 \%$ reduction in application runtime, LOCO CC+VMS results in further $4.8 \%$ reduction, and LOCO CC+VMS+IVR gives an additional $3.7 \%$ reduction. Overall, LOCO improves runtime by $13.9 \%$, on average, across all benchmarks. For 256-core CMP, as shown in Figure 11b, LOCO gives higher runtime reduction of $17.9 \%$, which shows scalability of LOCO to higher core counts.

Comparison to LOCO with Alternative NoC Architectures. So far, all simulations for both the baselines and LOCO run on top of SMART NoC. To demonstrate that LOCO's performance is hinged upon SMART NoC's capability to instantly establish a virtual, single-cycle multihop path, we turn to evaluation of LOCO with an alternative NoC architectures: conventional NoC and high-radix routers. When LOCO runs on top of conventional $\mathrm{NoC}$ and thus without VMS broadcasting, it is essentially reduced to the baseline clustered cache with increased network latency by stopping at every intermediate router.

High-Radix routers use the same clockless repeated wire technology as SMART, to go up to 4 hops within 1 cycle, and represent an alternate way of leveraging low wire delay. We implement a Flattened Butterfly topology [28] which adds dedicated physical links from every router to its 1hop, 2-hop, 3-hop and 4-hop neighbors to enable LOCO to run with $4 \times 4$ cluster sizes. Each home node is now always 

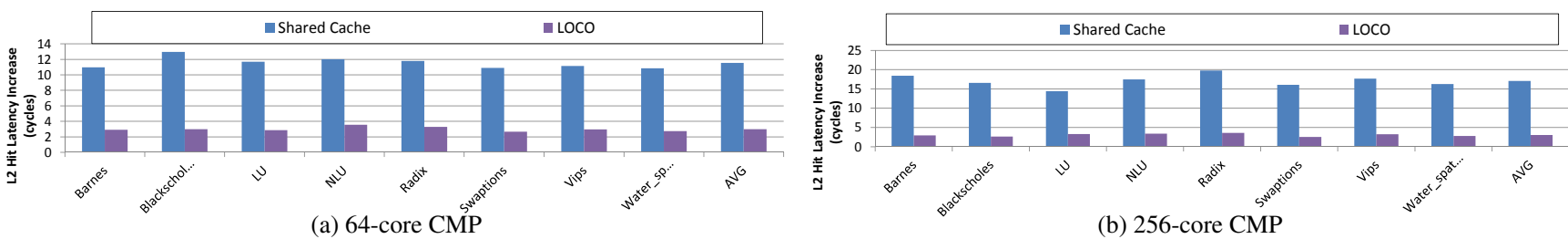

Figure 7: Increase of L2 access latency over Private Cache

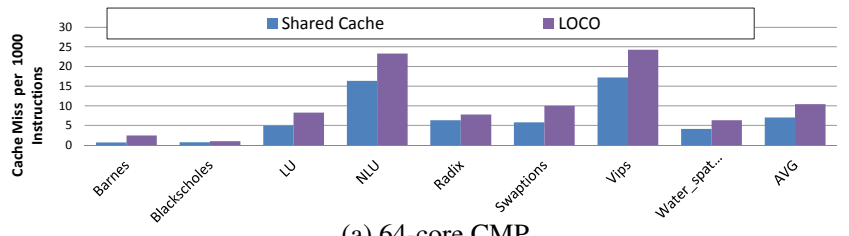

(a) 64-core CMP

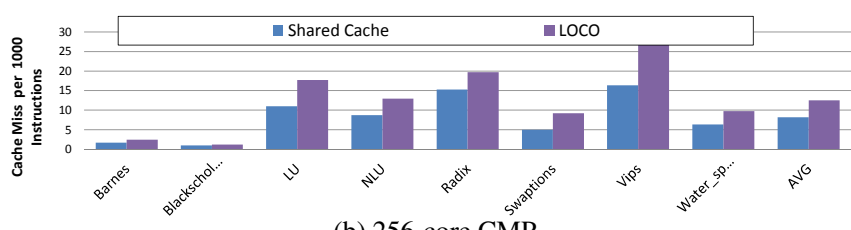

(b) 256-core CMP

Figure 8: L2 Cache miss per a thousand instructions according to cache configurations

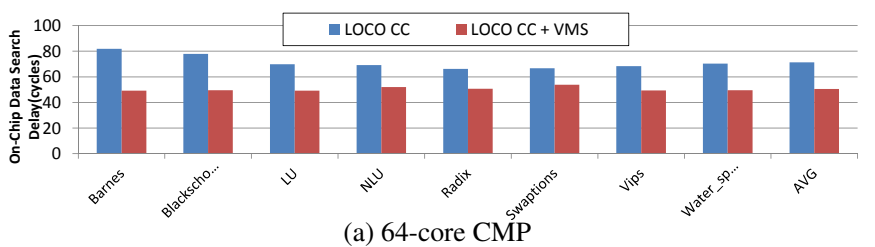

Figure 9: Global search delay for data cached on-chip

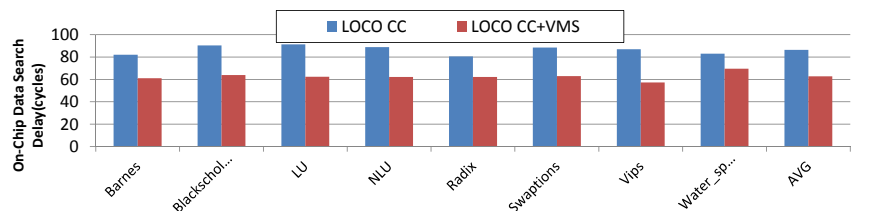

(b) 256-core CMP

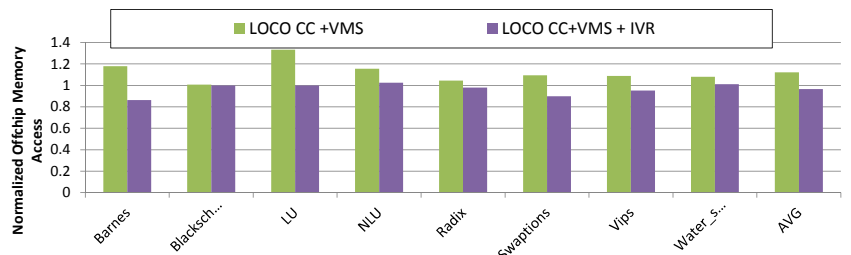

(a) 64-core $\mathrm{CMP}$

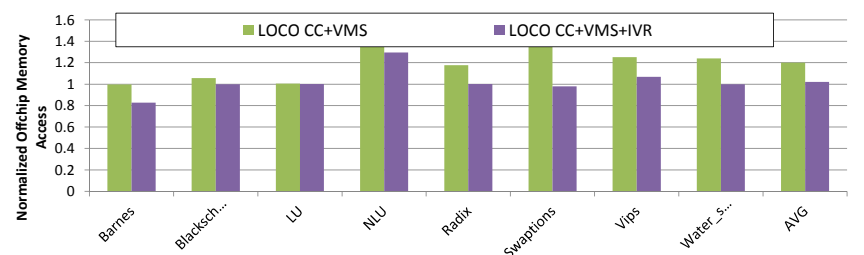

(b) 256-core CMP

Figure 10: Normalized off-chip memory access against baseline Shared Cache

1-cycle away unlike SMART where 1-cycle is opportunistic, and the network has $4 \mathrm{x}$ higher bisection throughput because of the additional wires. However, each router is now 20-ported instead of the 5-ported SMART/baseline router, which requires multi-stage arbiters and crossbars, increasing the router pipeline to at least 4-stages $[27,28,40]$ unlike the 2-stage SMART router. There is also a $6.7 \mathrm{X}$ area and $2.3 \mathrm{X}$ power overhead as compared to SMART by modeling both designs in DSENT [41].

Figure 12 shows that conventional $\mathrm{NoC}$ and high-radix routers increase both L2 access latency and global on-chip data search delay. For 256-core, the overhead by conventional NoC is 2.01x for L2 hit latency, and 1.99x for global on-chip data search delay, while the overhead by high-radix routers is 3.10x for L2 hit latency, and 1.59x for global onchip data search delay. Figure 13 shows the runtimes normalized against shared cache running atop SMART NoC. LOCO with SMART NoC reduces the runtime by $18.9 \%$ for 64 core, and by $24.6 \%$ for 256 -core , compared to LOCO with conventional NoC. The increased router latencies of highradix routers result in $22.7 \%$ runtime increase for 64 -core, and $24.4 \%$ increase for 256-core, compared to LOCO with SMART. We note that LOCO with high-radix routers underperform even LOCO with conventional NoC. This is because high-radix routers increases L2 access latency significantly within a cluster, since the request always go through 4-stage pipelined routers both at the source and at the destination.

Cluster Size and Topology. Till now we presented results with $4 \mathrm{x} 4$ clusters. Now, for 64 -core CMP, we explore the impact of alternative cluster sizes and topology: $4 \times 1$ and $8 \times 1$. Cluster sizes of $4 \times 1$ allow a SMART-hop distance of 1 for every pair of two nodes within the cluster, while cluster sizes of $8 \times 1$ and $4 \times 4$ have up to 2 SMART-hops. As shown Figure 14a, smaller cluster sizes lead to reduced L2 cache hit latencies; 1.17 and 0.45 cycles are reduced for cluster size $4 \times 1$ and $8 \times 1$, respectively. However, smaller cluster sizes lead to $35 \%$ and $20 \%$ higher L2 cache miss rates on average, as shown in Figure 14b. Cluster size should be chosen considering trade-off between L2 cache hit latency and miss rate. Figure 14d compares the runtimes of LOCO with different cluster sizes. The optimal cluster size and topology is dependent on application, since these trade-offs 


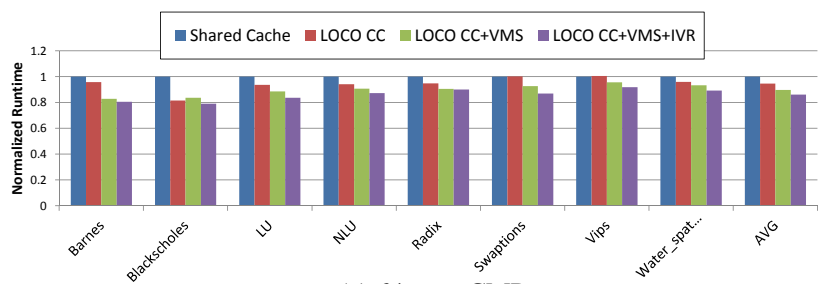

(a) 64-core CMP

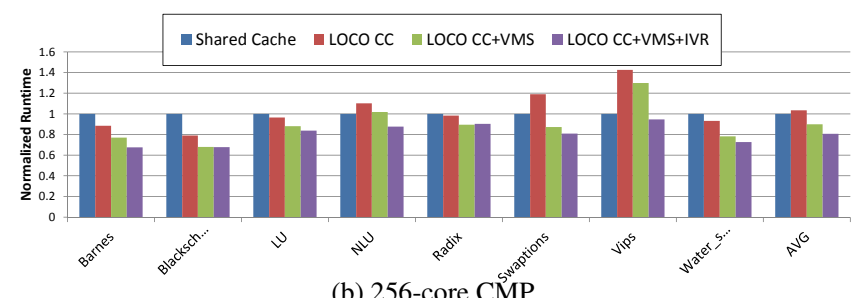

(b) 256-core CMP

Figure 11: Normalized runtimes of LOCO and Hybrid Caches against baseline Shared Cache

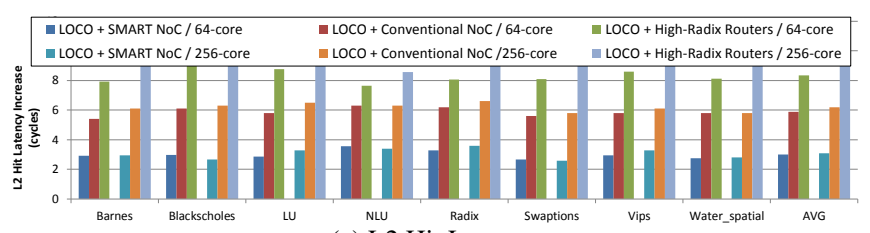

(a) L2 Hit Latency

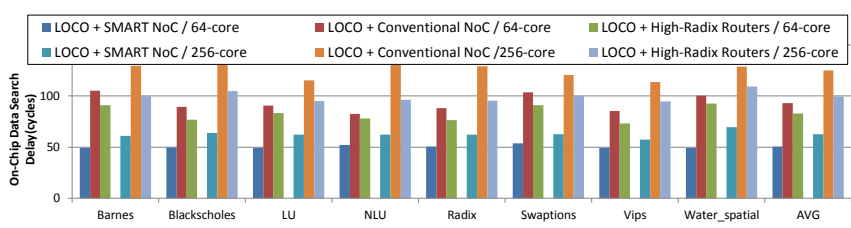

(b) Global on-chip Data Search Delay

Figure 12: Comparison to LOCO with Conventional NoC and with High-Radix routers: Memory Latency

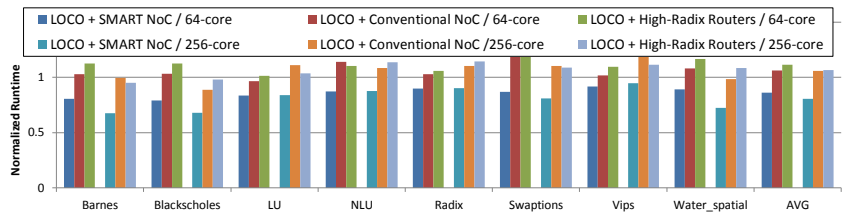

Figure 13: Comparison to LOCO with Conventional NoC and with High-Radix routers: Normalized Runtime

can have different impact on the performance according to application characteristic. For example, cluster size $4 \times 1$ has $89.9 \%$ higher cache miss rate for swaptions than cluster size $4 \times 4$, while the increase is mere $15.6 \%$ for water_spatial. As $^{2}$ a result, cluster size $4 \times 1$ has the worst runtime for swaptions, while it is the best for water_spatial.

Table 2: Multi-program workloads for 64-core CMP

\begin{tabular}{|c|c|}
\hline $\begin{array}{c}\text { Work- } \\
\text { load }\end{array}$ & $\begin{array}{l}\text { Description } \\
\text { Number of instances x Benchmark( Number of Threads ) }\end{array}$ \\
\hline W0 & 4xBlackscholes(4), 4xFerret(4), 4xFmm(4), 4xLu(4) \\
\hline W1 & 4xNlu(4), 4xSwaptions(4), 4xWater_Nsq(4), 4xWater_Spatial(4) \\
\hline $\mathrm{W} 2$ & 4xBlackscholes(4), 4xFerret(4), 4xWater_Nsq(4), 4xWater_Spatial(4) \\
\hline W3 & 4xFmm(4), 4xLu(4), 4xNlu(4), 4xSwaptions(4) \\
\hline W4 & 4xBlackscholes(4), 4xFerret(4), 4xNlu(4), 4xSwaptions(4) \\
\hline W5 & 2xBlackscholes $(8), 2 \times$ Ferret $(8), 2 \times F m m(8), 2 \times L u(8)$ \\
\hline W6 & 2xNlu(8), 2xSwaptions(8), 2xWater_Nsq(8), 2xWater_Spatial(8) \\
\hline W7 & 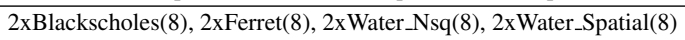 \\
\hline W8 & 1xBlackscholes(16), 1xFerret(16), 1xFmm(16), 1xLu(16) \\
\hline W9 & 1xNlu(16), 1xSwaptions(16), 1xWater_Nsq(16), 1xWater_Spatial(16) \\
\hline
\end{tabular}

Multi-program Workloads. The need for clustering in cache organization naturally occurs with mapping of heterogeneous tasks onto CMP, where the memory is shared mostly within the same task. Thus, local L2 caches of cores running the same task can be clustered into a shared cache to provide reduced L2 hit latency within the cluster, while it operates as private cache globally $[18,31,35]$. The resulting clustered cache is similar to LOCO CC, where the cluster size is determined by the number of threads(cores) required for individual task. One of important design goals in clustered cache is to allocate the cache resource among different tasks for high utilization of on-chip cache, and thus to reduce costly off-line memory access. Several previous works have proposed dynamic reallocation of cache resources adapted by memory demand of each task $[18,31]$, which, in turn, poses intriguing problem of managing the increased cost of global data search. LOCO can address this problem by inter-cluster victim replacement, which allows one cluster to utilize less utilized cache resource in other clusters across the chip.

In the evaluation, both the baseline clustered cache and LOCO provide shared cache within clusters. On the other hand, each task in the workloads is assumed to have exclusive address space with each other, thus the second level cache coherence is not required. The baseline clustered cache is modelled only to provide shared cache and cache coherence within the cluster. Atop the baseline clustered cache, LOCO performs inter-cluster victim replication with faster retrieval of migrated data on VMS in global scale. Table 2 summarizes the workloads used for the evaluation. While LOCO supports flexible heterogeneous clusters in which each cluster can have any size and can be changed at any time as depicted in Figure 1, we assume that all clusters are of the same size while running each workload for the ease of simulation; clustered cache and LOCO have cluster size $4 \mathrm{x} 1$ for W0 through W4, cluster size $8 \mathrm{x} 1$ for W5 through W8, and cluster size $4 \times 4$ for the rest. Each individual task is mapped onto a single cluster. For example, with the workload W0, every instance of tasks requires 4 threads, thus 64-core CMP is divided into 16 clusters of size $4 \times 1$. As there are 4 instances of barnes, 4 clusters run their own instance of barnes independently, another 4 clusters run blackscholes, and so on. For all cache organizations, the same SMART NoC with $H P C_{\max }=4$ is applied as usual. Figure 15 a shows off-chip memory access normalized against shared cache. On average, clustered cache increases off-line memory access by $26.6 \%$, while LOCO reduces off- 

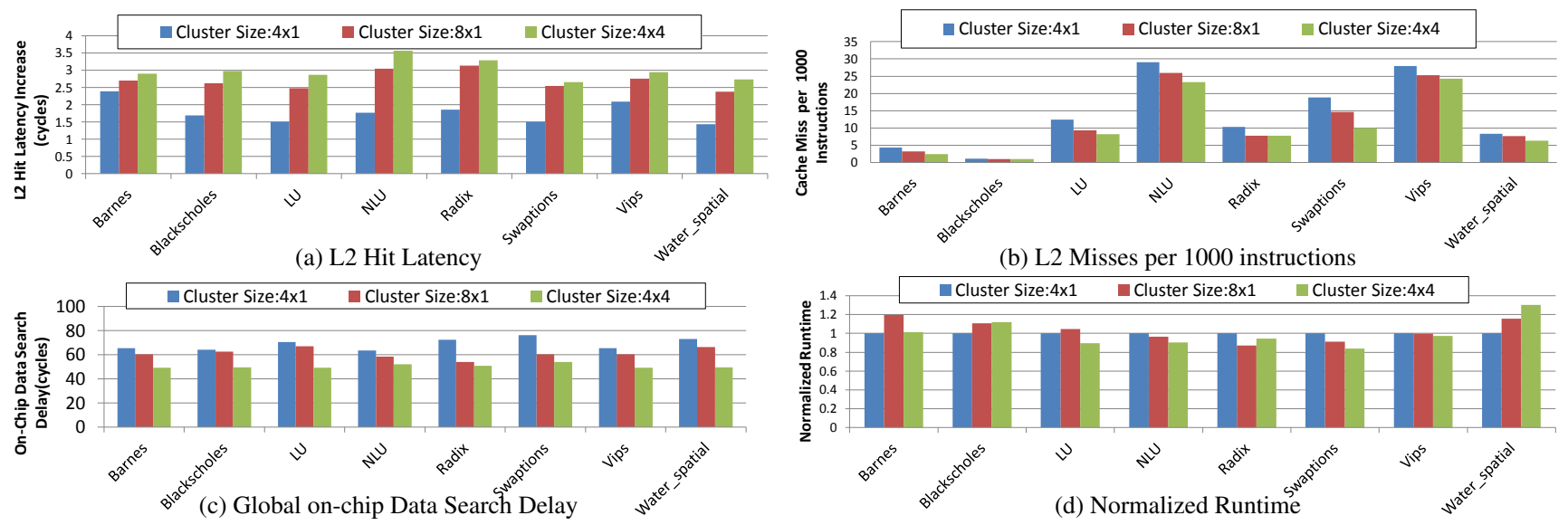

Figure 14: Performance of LOCO according to different cluster sizes
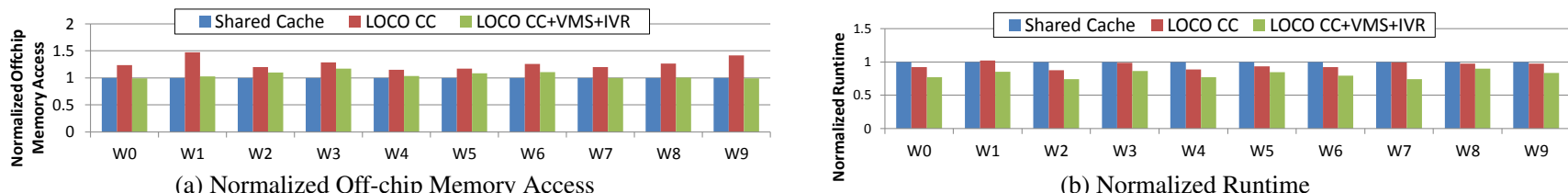

(a) Normalized Off-chip Memory Access

(b) Normalized Runtime

Figure 15: LOCO with multi-program workloads

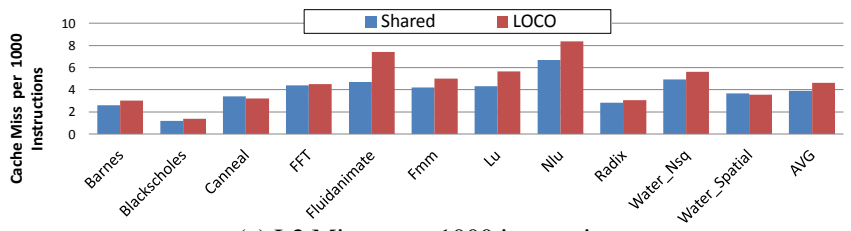

(a) L2 Misses per 1000 instructions

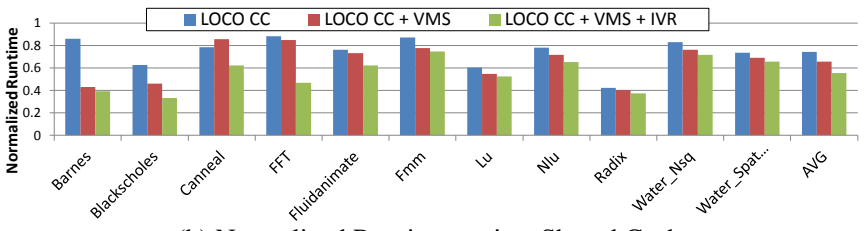

(b) Normalized Runtime against Shared Cache

Figure 16: Full system simulation of LOCO for 64-core CMP

line memory access to almost the same level as shared cache, just $5.1 \%$ higher than shared cache. As a result, Figure 15b shows that LOCO reduces the runtime of clustered cache by $13.8 \%$, which is $20 \%$ improvement over shared cache.

\subsection{Full System Simulations}

Trace-driven simulations do not properly capture dependencies between instructions that cause busy-waiting or spinning, which can have high performance impact. In this section, we present full system simulation results to evaluate the performance of LOCO more realistically. Due to tool limitation, we conducted full system simulations only for 64-core CMP. We leave verifying LOCO with full system simulations at high core counts as future work.

Figure 16b shows normalized runtimes for PARSEC and SPLASH-2 benchmarks ${ }^{8}$ against shared cache. On average, LOCO CC delivers $26 \%$ reduction in application runtime, LOCO CC+VMS further results in $8 \%$ reduction, and LOCO $\mathrm{CC}+\mathrm{VMS}+\mathrm{IVR}$ gives an additional $10 \%$ reduction. Overall,

\footnotetext{
${ }^{8}$ For full system simulations, we were unable to obtain results of swaptions and vips due to long simulation time. Instead, we added results of canneal, $f f t$, fmm fluidanimate, and water_nsq, which in turn, failed in trace-driven simulations due to out-of-memory errors.
}

LOCO improves runtime by $44.5 \%$ on average. We note that benchmarks such as blackscholes, lu, and radix, show significantly reduced runtime with just clustering. In contrast, barnes and fft are not improved much by only clustering. This is because the communication pattern in the first group is highly concentrated between neighboring cores, while the latter group exhibits widely distributed communication patterns across the die [5]. Although the latter benchmarks lack locality by nature, LOCO successfully reduces the runtimes of barnes with VMS, or fft with IVR, giving consistent performance improvement across the benchmarks.

\section{Related Work}

For the first time, SMART NoC provides a practical mechanism by which any core within $H_{\max }$ hops away can be reached in a single cycle, at any time. LOCO leverages this unique mechanism with adaptive cluster sizes, virtual meshes for inter-cluster broadcasts, and using remote nodes for storing victims. None of these make sense without SMART, and as we will next discuss, many prior cache organizations will work better with SMART, but none leverages SMART as well as LOCO. 
Locality-optimized data placement. There has been a plethora of recent studies that optimizes locality for higher performance. One such approach is the dynamic mapping of pages to L2 cache slices in a shared cache, often with the aid of the OS [3,10,14, 15, 19]. There has been significant work on improving data locality at the cache line granularity as well. Cache line is relocated closer to the core $[12,26]$, or replicated into the local L2 cache $[45,46]$, which can be further improved by selective replication [7, 13].

All these works will have to be reformulated to leverage SMART NoCs. For instance, with SMART, locality-aware mapping or replication need no longer be limited to a local L2 cache slice, but instead, can be expanded to all L2 caches within $H_{\max }$. Similarly, we can remove unnecessary redundancy by retaining only a single copy of data within a radius of $H_{\max }$. In fact, these are exactly the observations that motivated LOCO's clustering. However, unlike these previous approaches, LOCO is not only concerned with data locality, but also leverages SMART NoC to maximize cache utilization by way of inter-cluster broadcasts and victim replacement.

Clustered cache organizations. Hierarchical or clustered caches have been proposed as a scalable cache solution by many researchers. In [32, 42], the communication bottleneck to a central directory is mitigated by distributing hierarchical directories across the chip. More recent studies have focused on providing flexible sized clusters to best serve application demands [9, 18, 23, 31, 35]. LOCO provides an efficient mechanism for supporting flexible clusters while maintaining coherence and balancing cache utilization across clusters with IVR, unlike the complex hardware monitoring and remapping mechanisms required by prior techniques.

In this paper, we did not focus on the policies for determining the most appropriate cluster size, as this depends on the applications' working sets and/or aggregate cache requirement [18, 23, 31, 35]. To find optimal cluster size, LOCO can leverage previous works which flexibly adjust cluster sizes according to the cache performance [18, 23, 31].

Cache search and coherence. Substantial research has proposed flexible data location in L2 cache slices, which leads to the additional overhead of searching for the current data location. Here, we focus on search techniques that manages at the granularity of cache lines. One approach is to use a central tag to keep track of dynamic locations of each cache line $[9,12]$. As a central tag directory might be a communication bottleneck, several researchers employed local tag directories instead [13, 24, 35]. A local tag directory can only function as a backup, since it is impossible to assign to every core its own tag directory covering the entire on-chip cached memory. The most common strategy is to maintain the tag directory at static home node locations [19, 24, 31, 45, 46], while actual data can be stored at other places. This leads to an indirection cost to access the home node whenever accessing data.

To avoid this indirection cost, broadcast can be used [7, $12,13,18,26,35,36]$. However broadcasting over largescale CMPs is simply unacceptable in terms of latency and energy consumption. The broadcasting cost is mitigated by using local tag directories as caches $[13,35]$, or by broadcasting only to relevant destinations $[18,36]$, which is similar to our approach. In this paper, we further optimize broadcast latency with our virtual meshes (VMS) leveraging SMART NoC (Section 3.2), which offers a scalable solution to many-core CMPs.

Co-design of cache organization with novel interconnects. There have been prior studies that investigate alternative cache designs driven by emerging interconnect technologies as well. Transmission line caches [6] enable area and energy-efficient cache organization by exploiting onchip transmission lines which can substantially reduce wire delay at the cost of wire bandwidth density. ATAC [30] leverages on-chip optical links as a high-bandwidth, low-energy broadcast bus for scalable broadcast-based coherence. Like these prior works, LOCO is motivated by novel interconnects, SMART. However, LOCO is not limited to a specific topology of custom-designed links determined at the design time, due to the flexible nature of SMART NoC. LOCO can adapt to applications or programmers' needs, and thus tailor a cache organization to SMART characteristics so as to truly be locality-oblivious.

\section{Conclusion}

This work presents LOCO, a locality-oblivious cache organization scheme that leverages recent innovations in lowlatency NoC architectures to redefine the notion of locality. SMART NoCs enable flits to traverse up to $H P C_{\text {max }}{ }^{-}$ hops (4 in this design) within one cycle at $2 \mathrm{GHz}$. LOCO is a hybrid private-shared cache organization that partitions the chip into virtual clusters of varying sizes. It provides increased hit rate within each cluster with fast access latencies, and fast global data search across chip over virtual meshes. It also enables data migration across clusters to reduce offchip accesses. LOCO demonstrates better performance than both private and shared caches, and provides a scalable cache organization and management solution as we move towards 1000-core chips.

\section{Acknowledgments}

The authers acknowledge the support of Semiconductor Research Corporation (SRC) and the Defense Advanced Research Projects Agency (DARPA) through the Semiconductor Technology Advanced Research network (STARnet), under the Center for Future Architectures (C-FAR) research center. 


\section{References}

[1] N. Agarwal, T. Krishna, L.-S. Peh, and N. K. Jha. Garnet: A detailed on-chip network model inside a full-system simulator. In ISPASS, 2009.

[2] N. Agarwal, L.-S. Peh, and N. K. Jha. In-network snoop ordering (inso): Snoopy coherence on unordered interconnects. In $H P C A, 2009$.

[3] M. Awasthi, K. Sudan, R. Balasubramonian, and J. B. Carter. Dynamic hardware-assisted software-controlled page placement to manage capacity allocation and sharing within large caches. In HPCA, 2009.

[4] R. Balasubramonian, N. P. Jouppi, and N. Muralimanohar. Multi-Core Cache Hierarchies. Synthesis Lectures on Computer Architecture. Morgan \& Claypool Publishers, 2011.

[5] N. Barrow-Williams, C. Fensch, and S. W. Moore. A communication characterisation of splash-2 and parsec. In IISWC, 2009.

[6] B. M. Beckmann and D. A. Wood. TLC: Transmission Line Caches. In MICRO, 2003.

[7] B. M. Beckmann, M. R. Marty, and D. A. Wood. Asr: Adaptive selective replication for $\mathrm{cmp}$ caches. In MICRO, 2006.

[8] C. Bienia, S. Kumar, J. P. Singh, and K. Li. The parsec benchmark suite: characterization and architectural implications. In PACT, 2008.

[9] J. Chang and G. S. Sohi. Cooperative caching for chip multiprocessors. In ISCA, 2006.

[10] M. Chaudhuri. Pagenuca: Selected policies for page-grain locality management in large shared chip-multiprocessor caches. In HPCA, 2009.

[11] C.-H. O. Chen, S. Park, T. Krishna, S. Subramanian, A. P. Chandrakasan, and L.-S. Peh. SMART: A Single-Cycle Reconfigurable NoC for SoC Applications. In DATE, 2013.

[12] Z. Chishti, M. D. Powell, and T. N. Vijaykumar. Distance associativity for high-performance energy-efficient non-uniform cache architectures. In MICRO, 2003.

[13] Z. Chishti, M. D. Powell, and T. N. Vijaykumar. Optimizing replication, communication, and capacity allocation in cmps. In ISCA, 2005.

[14] S. Cho and L. Jin. Managing distributed, shared 12 caches through os-level page allocation. In MICRO, 2006.

[15] B. Cuesta, A. Ros, M. E. Gómez, A. Robles, and J. Duato. Increasing the effectiveness of directory caches by deactivating coherence for private memory blocks. In ISCA, 2011.

[16] N. Eisley, L.-S. Peh, and L. Shang. Leveraging on-chip networks for data cache migration in chip multiprocessors. In PACT, 2008.

[17] A. Gupta, W.-D. Weber, and T. C. Mowry. Reducing memory and traffic requirements for scalable directory-based cache coherence schemes. In ICPP (1), 1990.

[18] M. Hammoud, S. Cho, and R. G. Melhem. Dynamic cache clustering for chip multiprocessors. In ICS, 2009.

[19] N. Hardavellas, M. Ferdman, B. Falsafi, and A. Ailamaki. Reactive nuca: near-optimal block placement and replication in distributed caches. In ISCA, 2009.
[20] R. Ho. On-Chip Wires: Scaling and Efficiency. PhD thesis, Stanford University, 2003.

[21] Y. Hoskote, S. R. Vangal, A. Singh, N. Borkar, and S. Borkar. A 5-ghz mesh interconnect for a teraflops processor. IEEE Micro, 27(5):51-61, 2007.

[22] J. Howard, S. Dighe, S. R. Vangal, G. Ruhl, N. Borkar, S. Jain, V. Erraguntla, M. Konow, M. Riepen, M. Gries, G. Droege, T. Lund-Larsen, S. Steibl, S. Borkar, V. K. De, and R. F. V. der Wijngaart. A 48-Core IA-32 Message-Passing Processor with DVFS in 45nm CMOS. In ISSCC, 2010.

[23] J. Huh, C. Kim, H. Shafi, L. Zhang, D. Burger, and S. W. Keckler. A nuca substrate for flexible cmp cache sharing. In ICS, 2005.

[24] N. D. E. Jerger, L.-S. Peh, and M. H. Lipasti. Virtual tree coherence: Leveraging regions and in-network multicast trees for scalable cache coherence. In MICRO, 2008.

[25] B. Kim and V. Stojanovic. Characterization of equalized and repeated interconnects for noc applications. IEEE Design and Test of Computers, 25:430-439, 2008.

[26] C. Kim, D. Burger, and S. W. Keckler. An adaptive, nonuniform cache structure for wire-delay dominated on-chip caches. In ASPLOS, 2002.

[27] J. Kim, W. J. Dally, B. Towles, and A. K. Gupta. Microarchitecture of a high-radix router. In ISCA, 2005.

[28] J. Kim, W. J. Dally, and D. Abts. Flattened butterfly: a costefficient topology for high-radix networks. In ISCA, 2007.

[29] T. Krishna, C.-H. O. Chen, W. C. Kwon, and L.-S. Peh. Breaking the on-chip latency barrier using SMART. In HPCA, 2013.

[30] G. Kurian, J. E. Miller, J. Psota, J. Eastep, J. Liu, J. Michel, L. C. Kimerling, and A. Agarwal. ATAC: All-to-All Computing Using On-Chip Optical Interconnects. In BARC, 2007.

[31] H. Lee, S. Cho, and B. R. Childers. Cloudcache: Expanding and shrinking private caches. In HPCA, 2011.

[32] Y.-C. Maa, D. K. Pradhan, and D. Thiébaut. A hierarchical directory scheme for large-scale cache-coherent multipmcessors. In IPPS, 1992.

[33] M. M. K. Martin, M. D. Hill, and D. A. Wood. Token coherence: Decoupling performance and correctness. In ISCA, 2003.

[34] M. M. K. Martin, D. J. Sorin, B. M. Beckmann, M. R. Marty, M. Xu, A. R. Alameldeen, K. E. Moore, M. D. Hill, and D. A. Wood. Multifacet's general execution-driven multiprocessor simulator (gems) toolset. SIGARCH Computer Architecture News, 33(4):92-99, 2005.

[35] M. R. Marty and M. D. Hill. Virtual hierarchies to support server consolidation. In ISCA, 2007.

[36] M. R. Marty, J. D. Bingham, M. D. Hill, A. J. Hu, M. M. K. Martin, and D. A. Wood. Improving multiple-cmp systems using token coherence. In HPCA, 2005.

[37] J. E. Miller, H. Kasture, G. Kurian, C. G. III, N. Beckmann, C. Celio, J. Eastep, and A. Agarwal. Graphite: A distributed parallel simulator for multicores. In HPCA, 2010.

[38] S. Park, T. Krishna, C.-H. Chen, B. Daya, A. Chandrakasan, and L.-S. Peh. Approaching the theoretical limits of a mesh 
NoC with a 16 -node chip prototype in $45 \mathrm{~nm}$ SOI. In $D A C$, 2012.

[39] K. Sankaralingam, R. Nagarajan, H. Liu, C. Kim, J. Huh, D. Burger, S. W. Keckler, and C. R. Moore. Exploiting ilp, tlp, and dlp with the polymorphous trips architecture. IEEE Micro, 23(6):46-51, 2003.

[40] S. Scott, D. Abts, J. Kim, and W. J. Dally. The BlackWidow high-radix clos network. In ISCA, 2006.

[41] C. Sun, C.-H. O. Chen, G. Kurian, L. Wei, J. Miller, A. Agarwal, L.-S. Peh, and V. Stojanovic. DSENT - a tool connecting emerging photonics with electronics for opto-electronic networks-on-chip modeling. In NoCS, 2010.

[42] D. A. Wallach. PHD: A Hierarchical Cache Coherent Protocol. MS Thesis. MIT, 1992.
[43] D. Wentzlaff, P. Griffin, H. Hoffmann, L. Bao, B. Edwards, C. Ramey, M. Mattina, C.-C. Miao, J. F. B. III, and A. Agarwal. On-chip interconnection architecture of the tile processor. IEEE Micro, 27(5):15-31, 2007.

[44] S. C. Woo, M. Ohara, E. Torrie, J. P. Singh, and A. Gupta. The splash-2 programs: Characterization and methodological considerations. In ISCA, 1995.

[45] M. Zhang and K. Asanovic. Victim migration: Dynamically adapting between private and shared $\mathrm{cmp}$ caches. Technical report, MIT, 2005.

[46] M. Zhang and K. Asanovic. Victim replication: Maximizing capacity while hiding wire delay in tiled chip multiprocessors. In ISCA, 2005. 